\title{
MEDIACIÓN TRANSNACIONAL: UNA APROXIMACIÓN PRÁCTICA EN EL ÁMBITO DE LAS RELACIONES PRIVADAS INTERNACIONALES
}

\author{
TRANSNATIONAL MEDIATION: A PRACTICAL APPROACH IN THE \\ TREATMENT OF PRIVATE INTERNATIONAL RELATIONSHIPS
}

\author{
Carmelo Hernández Ramos \\ Universidad de Alicante. España/Spain \\ carmelo.hernandez@ua.es
}

Recibido/Received: 18/02/2015

Modificado/Modified: 14/04/2015

Aceptado/Accepted: 27/04/2015

\section{RESUMEN}

La resolución de conflictos a través de la mediación, en el ámbito de las relaciones privadas internacionales y la mediación intercultural, en particular, aquella que se realiza con personas de otros países o culturas, requiere de sólidas herramientas de gestión de conflictos, y de eficientes habilidades de negociación. En la resolución de conflictos en el ámbito civil, familiar, comercial y, en general, privado internacional, son necesarias estrategias y habilidades específicas de mediación para poder resolver conflictos en los que hay que abordar aspectos interculturales y jurídicos, junto a otros de naturaleza psicológica y sociocultural. ¿Por qué utilizar entonces la mediación como herramienta de solucionar un conflicto en este complejo ámbito? Porque se pretende solucionar disputas evitando largos y costosos procedimientos para las partes, lo que conlleva la incorporación de técnicas de gestión de conflictos, junto a habilidades de negociación y estrategias de comunicación y solución extrajudicial de los conflictos planteados.

\section{PALABRAS CLAVE}

Mediación, interculturalidad, inmigración, relaciones privadas, ciudadanía inclusiva.

\section{SUMARIO}

1. Introducción. 2. Mediación y ADR. 3. La mediación transfronteriza. 4. La gama de grises. Bibliografía.

\begin{abstract}
Conflict resolution through mediation, in the course of private international relationships and in the context of multiculturalism, particularly when it involves individuals from other countries or cultures requires robust tools for conflict management and efficient negotiation skills. In the resolution of civil or commercial conflicts and, in general, in the camp of international private mediation, is necessary to know and apply strategies and specific skills to resolve conflicts that must be addressed having in mind his legal aspects, along with his interdisciplinary characteristics and specially his intercultural nature. Why then is good to use mediation as a tool to solve a conflict in this so complex area? Because it aims to solve disputes avoiding lengthy and costly procedures for the persons, which involves the incorporation of conflict management techniques, together with negotiation skills and communication strategies and extrajudicial settlement of disputes raised.
\end{abstract}

\section{KEYWORDS}

Mediation, interculturalism, immigration, private relationships, inclusive citizenship. 


\section{CONTENTS}

1. Introduction. 2. Mediation and ADR. 3. Cross-border mediation. 4. Shades of gray. References.

\section{INTRODUCCIÓN}

La resolución de conflictos a través de la mediación, dedicando especial atención a la mediación en asuntos civiles y mercantiles en el ámbito de las relaciones privadas internacionales y a la mediación intercultural, en particular, aquella que se realiza con personas de otros países o culturas, requiere de sólidas herramientas de gestión de conflictos, $\mathrm{y}$ de eficientes habilidades de negociación.

En la resolución de conflictos en el ámbito civil, familiar, comercial y, en general, privado internacional, son necesarias estrategias que, en esta difícil materia, cualifiquen y distingan, las buenas prácticas de las que no lo son. La formación del mediador es y debe ser, por tanto, fundamental para poder resolver conflictos de esta naturaleza, pues en su praxis mediatoria deberá considerar y abordar aspectos interculturales y jurídicos, junto a otros de naturaleza psicológica y sociocultural. Necesitará, por tanto, utilizar herramientas prácticas eficaces para la resolución de la controversia o problema, que deberán tener en cuenta aspectos tan dispares como el coste de oportunidad del acuerdo, las barreras lingüísticas o la discreción en la visibilidad de su trabajo, por mencionar solo unos pocos.

¿Por qué utilizar la mediación como herramienta de solucionar conflictos en este complejo ámbito? La respuesta es clara, porque se pretende solucionar la disputa evitando un procedimiento arbitral o judicial largo y costoso para las partes. En este ámbito tan complejo, queda claro que los mediadores y mediadoras no solo han de incorporar en su actividad específica técnicas de gestión de conflictos, sino también han de saber desarrollar y poner en acción habilidades de negociación y estrategias de comunicación y solución extrajudicial de los conflictos planteados.

\section{MEDIACIÓN Y ALTERNATIVE DISPUTE RESOLUTION (ADR)}

Todas las sociedades, comunidades y organizaciones afrontan conflictos en diferentes oportunidades y situaciones. Las disputas existen cuando las personas compiten para alcanzar objetivos que aparentemente son o pueden ser incompatibles. Las personas que afrontan conflictos, a menudo necesitan ayuda externa para resolver sus controversias. A un nivel macroscópico, la cooperación se está convirtiendo en la clave de la supervivencia humana. La cooperación no significa necesariamente el fin de la controversia o disputa, sino la posibilidad de abordarla más constructivamente. Es el camino que lleva del enfrentamiento a la cooperación y tiene un nombre propio: mediación.

La mediación, sin embargo, es un procedimiento no adversarial de resolución de controversias mediante el cual las partes en conflicto son realmente los protagonistas principales de la construcción del iter que conduce a la pacificación de la disputa origen del problema, ya que no delegan la resolución del mismo en una tercera persona para que decida por ellas, sino que el poder de decisión lo retienen las mismas personas afectadas.

La mediación es flexible, en el sentido de que el proceso no sigue un orden procedimental rígido, sujeto a condiciones y plazos predeterminados y es voluntario porque las personas ingresan al mismo por propia decisión: ellas mismas determinan cual es la información que 
transmiten u ocultan; deciden si llegan o no a un acuerdo y se retiran cuando ellas así lo estiman conveniente o en el preciso instante en que dejen de sentirse cómodas: en una mediación, nadie está obligado a permanecer en contra de su voluntad. Consecuentemente, los acuerdos que se logran tienen una gran probabilidad de éxito, debido a la consistencia interna que aporta la gran carga emocional que poseen, al haber sido gestados en conjunto, por ambas partes. El proceso de mediación es confidencial, pues se concreta en un acuerdo, que suscriben los mediados, donde se establecen las reglas que deben satisfacer conjuntamente, garantizándose que nada de lo que allí se concrete pueda ser extrapolado, en ningún sentido, fuera del exclusivo ámbito que determina intrínsecamente el propio proceso de mediación. Tampoco el mediador podrá reproducir nada de lo que en el proceso se diga, ni ser citado como testigo, pues lo ampara el secreto profesional.

En este sentido, distinguimos, conforme a las Directrices de Naciones Unidas para una mediación eficaz, (anexo del informe del Secretario General sobre el fortalecimiento de la función de mediación en el arreglo pacífico de controversias, la prevención de conflictos y su solución -A/66/811, 25 de junio de 2012-); entre imparcialidad y neutralidad, entendiendo que la primera impone una labor mediadora evidentemente equidistante de las partes y sin influencia de prejuicios, juicios de valor o simpatías, pero con la presencia de determinados valores o principios que implican que no cualquier acuerdo es deseable, sino el que es mínimamente "justo" para ambas partes, y no consagra una desigualdad manifiesta entre ambas que sólo resolvería el conflicto con la cesión descompensada de los intereses y legítimos derechos de una de las partes.

Desde esta perspectiva, la mediación puede suponer no solo la consecución del acuerdo, sino también la reducción de la rivalidad entre las partes y la mejoría de la calidad en las relaciones interpersonales de las personas mediadas. Estos dos elementos potencian activamente la comunicación inter-partes posterior a la consecución del acuerdo y posibilitan no solo la evolutiva modificación de las correlativas percepciones de los mediados acerca del conflicto, sino que además contribuyen a acercar las posiciones más extremas en su inicial posicionamiento respecto del problema y su eventual solución. A tal fin, el mediador ha de saber poner en funcionamiento su pensamiento creativo y su inteligencia emocional, para poder obtener un número suficiente de soluciones posibles, alternativas y satisfactorias, respecto de aquellas que los mediados puedan percibir, desde sus posiciones de confrontación inicial, potenciando su propia capacidad de negociación y tomando como criterio y referencia sus verdaderos intereses y necesidades. En su actuación profesional, el mediador deberá facilitar la comunicación entre las partes, así como desarrollar una conducta activa para buscar el acercamiento de las posturas de los mediados. Aunque el mediador podrá renunciar a su labor, siempre y en todo caso debe preservar su sigilo profesional respecto de las personas, hechos y conocimientos adquiridos sobre los mismos.

Es en la Ley 5/2012, de 6 de julio, de mediación en asuntos civiles y mercantiles, en cuya Exposición de Motivos se indica que "La mediación está construida en torno a la intervención de un profesional neutral que facilita la resolución del conflicto por las propias partes, de una forma equitativa, permitiendo el mantenimiento de las relaciones subyacentes y conservando el control sobre el final del conflicto". En esta propia Ley (Artículo 1) se establece que "Se entiende por mediación aquel medio de solución de controversias, cualquiera que sea su denominación, en que dos o más partes intentan voluntariamente alcanzar por sí mismas un acuerdo con la intervención de un mediador (...), la figura del mediador es, (...) la pieza esencial del modelo, puesto que es quien ayuda a encontrar una solución dialogada y voluntariamente querida por las partes. La actividad de mediación se 
despliega en múltiples ámbitos profesionales y sociales, requiriendo habilidades que en muchos casos dependen de la propia naturaleza del conflicto. El mediador ha de tener, pues, una formación general que le permita desempeñar esa tarea y sobre todo ofrecer garantía inequívoca a las partes por la responsabilidad civil en que pudiese incurrir". Por lo tanto, es probable que, según la naturaleza de la mediación (comunitaria, civil, penal...), sea más apropiado un tipo u otro de perfil profesional específico junto a esa formación genérica que debe tener todo mediador.

Cabe destacar, igualmente, que el Título III de la referida Ley 5/2012 está dedicado al Estatuto del Mediador, recogiéndose, para este ámbito de la mediación civil y mercantil, que pueden ser mediadores las personas naturales que se hallen en pleno ejercicio de sus derechos civiles, siempre que no se lo impida la legislación a la que puedan estar sometidos en el ejercicio de su profesión. En los casos en que la mediación se haga conforme a la legislación española (Art. 2, Ley 5/2012), será referencia obligada el referido Título III de la Ley.

Respecto de las personas jurídicas que se dediquen a la mediación, la Ley indica que deberán designar para su ejercicio a una persona natural que reúna los requisitos previstos en la Ley.

Sobre la formación mínima acreditable, se indica que el mediador deberá estar en posesión de título oficial universitario o de formación profesional superior y además deberá contar con formación específica para ejercer la mediación, (que, se establece, se deberá adquirir mediante la realización de uno o varios cursos específicos impartidos por instituciones debidamente acreditadas).

Se contempla la sujeción a responsabilidad del mediador en el ejercicio de su labor, cumpliendo fielmente el encargo mediatorio, y por ello se establece que deberá suscribir un seguro o garantía equivalente que cubra la responsabilidad civil derivada de su actuación en los conflictos en que intervenga.

Los Mecanismos Alternativos de Resolución de Conflictos o Alternative Dispute Resolution (ADR), han alcanzado en nuestros días una manifiesta visibilidad en el ámbito de las relaciones privadas internacionales. Entre estos procedimientos encontramos el arbitraje y la mediación. En el primer caso, es un árbitro quien resolverá la controversia dictando un laudo que, por lo general, tendrá un carácter vinculante. En la mediación, un tercero neutral, investido de poder de dirección, sin capacidad para decidir, ayudará a las partes a que lleguen a un acuerdo vinculante mediante un procedimiento estructurado y flexible: el procedimiento de mediación.

Es importante destacar las diferencias existentes entre ambos mecanismos de resolución alternativa de conflictos: Arbitraje y mediación no son lo mismo en absoluto.

El arbitraje suele ser conceptuado como la "intervención en una disputa de un tercero independiente al que se le da la autoridad para recoger información, escuchar a ambas partes y tomar una decisión acerca de cómo resolver el conflicto". El arbitraje puede ser obligatorio o consultivo, según que la decisión final sea vinculante o no y por tanto resuelva definitivamente o no el conflicto.

Se dice también que se basa en la acción de discernimiento y de regulación de conflictos, sobre la base de una normativa preestablecida que prevé la capacidad de arbitrar de una persona o institución determinada y constituida con esta finalidad. En éste contexto, el árbitro se limita a aplicar las normas fijadas por la autoridad competente o por los acuerdos genéricos establecidos inicialmente, aunque procura la neutralidad y la corrección desde la presión disuasoria que representa la aplicación de sanciones o la transferencia a las autoridades judiciales competentes. Por ello, se trata aquí de un tercero con poder de decisión, independiente y que no compromete al sistema legal. 
A diferencia del mediador, el árbitro, luego de escuchar a las partes y recibir los antecedentes y pruebas, toma las decisiones por las partes (a diferencia de lo que ocurre en la mediación, donde la decisión queda en poder finalmente de las partes, no del mediador). Un ejemplo clásico: "Cuando dos alumnos/as que tienen un conflicto recurren al profesor/a para que defina qué hacer para solucionar el conflicto, los alumnos/as deben acatar la respuesta que dé el profesor/a".

Por lo tanto, la diferencia con la mediación se resume en que la mediación pone el énfasis en la participación voluntaria de las partes en el proceso y en la posible consecución del acuerdo final, mientras que en el arbitraje la resolución de la controversia es provista no por las parte, sino por un tercero.

Así, el arbitraje suele resultar eficaz en conflictos en los cuales no se han desarrollado perturbaciones emocionales y en los cuales se busca una solución más rápida y eficaz que el acudir a los Tribunales ordinarios. Sobre todo en disputas mercantiles es donde el arbitraje resulta más idóneo, no así en los conflictos en los que el elemento emocional pueda ejercer una influencia notable.

Los ADR se enmarcan dentro de los denominados movimientos de reforma para garantizar el acceso a la Justicia. En estos movimientos se han identificado tres etapas sucesivas en su evolución.

1.- Una primera etapa, identificada con el proveer servicios jurídicos para los más desfavorecidos o personas con menos recursos económicos, mediante el establecimiento, de sistemas de asistencia jurídica gratuita o servicios de abogados de oficio.

2.- La segunda etapa se identificaría con la articulación de figuras tales como las acciones populares y de grupo, como mecanismos para extender el acceso a la justicia a la protección de los intereses difusos y de los derechos colectivos.

3.- La tercera etapa se orienta a garantizar la efectividad del acceso a la justicia para la resolución de conflictos, bien sea a través de la justicia formal, mediante vías judiciales realmente expeditas o de procedimientos alternativos como la negociación, el arbitraje o la mediación.

Específicamente, los sistemas alternativos de resolución de conflictos (ADR) se inscriben dentro de las políticas sobre la mejora del acceso a la justicia, desempeñando un papel complementario en relación con los procedimientos jurisdiccionales, en la medida en que en muchos casos, los métodos aplicados en los sistemas alternativos de resolución de conflictos (ADR) pueden permitir a las partes entablar un diálogo, que de otro modo hubiera sido imposible entablar, y evaluar por sí mismas la conveniencia de dirigirse o no a los Tribunales.

Históricamente, la justicia restaurativa surgió en la década de los años 70 como una forma de mediación entre víctimas y delincuentes y en la década de los años 90 amplió su alcance para incluir también a las comunidades de apoyo, con la participación de familiares y amigos de las víctimas y los delincuentes en procedimientos de colaboración denominados "reuniones de restauración" y "círculos". Este nuevo enfoque en el proceso de subsanación para las personas afectadas por un delito y la obtención de control personal asociado parece tener un gran potencial para optimizar la cohesión social en nuestras sociedades cada vez más indiferentes. La justicia restaurativa y sus prácticas emergentes constituyen una nueva y promisoria área de estudio para las ciencias sociales.

El postulado fundamental de la justicia restaurativa es, concluyendo, que el delito perjudica a las personas y las relaciones sociales, y que la justicia necesita la mayor subsanación del daño ocasionado posible. 


\section{LA MEDIACIÓN TRANSFRONTERIZA}

El Art. 3 de la Ley 5/2012 se refiere de forma concreta a la mediación en conflictos transfronterizos, indicando que "un conflicto es transfronterizo cuando al menos uno de los partes tiene su domicilio o reside con habitualidad en un Estado distinto de aquel en que cualquiera de las otras partes a las que afecta la disputa, estén domiciliadas cuando acuerden hacer uso de la mediación, o bien sea obligatorio acudir a la misma, conforme a la Ley que resulte de aplicación". Hay que dejarlo bien claro: La Ley 5/2012, de 6 de julio, se aplica tanto a la mediación en la que no existe ningún elemento o característica de extranjería, como a aquella otra de carácter internacional, en la que existe algún elemento (al menos uno) de extranjería, incluyéndose en este perfil claramente a la mediación transnacional.

La mediación transfronteriza puede tener lugar especialmente en asuntos civiles y mercantiles referidos tanto a los particulares, como a las empresas. En los conflictos empresariales transfronterizos, la mediación tiene lugar en el ámbito de las relaciones privadas internacionales donde muchas veces intervienen diferentes leyes, idiomas y culturas, todo lo cual supone una barrera importante a la hora de afrontarlos. Puede evitar el excesivo coste económico que supondrían los desplazamientos y estancias en otro país, así como la contratación de abogados o los costes judiciales si se emprendieran acciones en los juzgados autóctonos; así como el coste temporal que suponen las dilaciones que se producen cuando se utilizan las vías tradicionales de resolución de conflictos.

Específicamente, esta mediación tiene lugar en el ámbito de las relaciones privadas internacionales, donde los conflictos y los litigios transfronterizos son más complejos debido a las diferentes leyes y jurisdicciones nacionales de cada país. Tal y como se especifica la Ley 5/2012 de mediación en asuntos civiles y mercantiles, en su Artículo 3, referido a la mediación en conflictos transfronterizos:

1) Un conflicto es transfronterizo cuando al menos una las partes está domiciliada o reside habitualmente en un Estado distinto a aquél en que cualquiera de las otras partes a las que afecta estén domiciliadas cuando acuerden hacer uso de la mediación o sea obligatorio acudir a la misma de acuerdo con la ley que resulte aplicable.

2) En los litigios transfronterizos entre partes que residan en distintos Estados miembros de la Unión Europea, el domicilio se determinará de conformidad con los artículos 59 y 60 del Reglamento (CE) n. ${ }^{\circ}$ 44/2001 del Consejo, de 22 de diciembre de 2000, relativo a la competencia judicial, el reconocimiento y la ejecución de resoluciones judiciales en materia civil y mercantil.

Aunque existen elementos que siempre pivotan en el lado de las dificultades (el coste final, las barreras lingüísticas o la discreción en la visibilidad de su trabajo, por mencionar solo unos pocos), son muchas las ventajas que apreciamos en la aplicación de la mediación transfronteriza:

a) La solución está hecha por las personas físicas o jurídicas mediadas, a la medida de sus propios intereses.

b) El procedimiento suele ser bastante más económico y rápido y elude las incertidumbres asociadas al eventual derecho, sentencia o juez de fondo.

c) La rigidez de la interlocución en sede judicial cede ante la apertura de la escucha activa y la tolerancia de la mediación.

d) El mediador en controversias internacionales es conocedor de las diferentes culturas que representan las personas mediadas y facilita la cooperación intercultural para crear un clima positivo de diálogo constructivo. 
e) El efecto mediación, consecuencia directa a medio y largo plazo, de la experiencia fruto de la participación en el proceso de mediación, potencia la inteligencia emocional de las personas y previene la resolución de futuras controversias y litigios de forma consensuada.

No puede haber mediación transfronteriza si no hay mediadores internacionales. Para poder ejercer tal profesión en nuestro país, hay que satisfacer los requisitos establecidos en el RD 980/2013, de 13 de Diciembre de 2013, de desarrollo de la Ley 5/2012. El mediador transnacional no ha de ser necesariamente un abogado o tener conocimientos jurídicos. Lo esencial es que tenga un entrenamiento y experiencia como mediador cualificado. Este terreno está abonado a la acción interdisciplinaria de la realidad visible en la interculturalidad presente en las relaciones privadas internacionales: cada persona puede tener un derecho original de referencia, proceder de una sociedad caracterizada por unas coordenadas sociales y demográficas determinadas, tener una especial personalidad y una forma de representar sus creencias y valores..., en suma: el mediador transnacional deberá disponer de una perfilación en su formación, habilidades y recursos que le habiliten para poder intervenir de forma eficiente, superando con su intervención las enormes dificultades e incertidumbres que van aparejadas a la celebración de un juicio internacional, en términos económicos, bioenergéticos, emocionales y personales. Es por ello imperativo que los medidores deben subordinar su actuación profesional a los códigos de conducta, controles de calidad y códigos de buenas prácticas establecidos en los respectivos ordenamientos jurídicos bajo los cuales desarrollen su praxis profesional.

El ámbito de aplicación de la Ley 5/2012 queda conformado del siguiente modo (Art.2):

1) En todo caso las mediaciones a practicar corresponderán al ámbito civil y mercantil, con independencia de su carácter nacional o transfronterizo.

2) Solo podrá afectar a derechos y obligaciones que estén a disposición de las partes conforme a lo establecido en las normas de conflicto que resulten aplicables, normas que determinarán cual es la ley que debe ser tenida en cuenta en cada caso.

3) Las personas o partes mediadas deberán aceptar y someterse, por tanto, de forma expresa o tácita a la Ley 5/2012, y en defecto de sometimiento expreso, al menos una de las partes debe tener su domicilio en territorio español, debiéndose realizar la mediación en España.

En la mediación transfronteriza o internacional son necesarios los siguientes requisitos metodológicos:

a) Un contrato de mediación y confidencialidad, que básicamente es un contrato de prestación de servicio, que deberá contener las reglas básicas de la mediación. Deberá ser firmado por el propio mediador o mediadora y las partes mediadas y demás personas intervinientes e le proceso de mediación.

b) El contrato compromete al mediador a prestar los servicios propios de la mediación y obliga a las partes mediadas al pago de los servicios prestados por el mediador o mediadora.

c) Las partes pueden llegar a un acuerdo de mediación sin aplicar ley alguna, puesto que no es necesario aplicar en la mediación ninguna norma jurídica, con las siguientes limitaciones:

i) La materia objeto de la controversia debe estar disponible para la mediación.

ii) El acuerdo al que se llegue en resolución de la disputa no debe ser contrario a Derecho, para determinar lo cual se tendrá en cuenta el Derecho que resulta aplicable según las normas de conflicto. El acuerdo, por tanto, no podrá ir en contra del orden público (Art. 12.3 CC). 
El proceso de mediación, flexible y a la vez estructurado, aparece regulado en el Título IV de la Ley 5/2012. Prácticamente consiste en un camino que empieza en la fase de premediación (preparatoria) y pro-mediación o mediación-en-sí-misma.

En la primera fase es importante señalar la siempre pertinencia de utilizar instrumentos científicos de recogida de información (entrevista cognitiva semiestructurada) para organizar bien "el mapa de la mediación".

Posteriormente se da paso a la mediación propiamente, que podríamos resumir en el siguiente "tren-de-lavado":

I) Fase inicial (Speech), que incluye la sesión constitutiva y el inicial speech o discurso inicial del mediador o mediadora, al que sigue la firma del acta constitutiva por todos los presentes en la sesión.

- Fase de exposición (Intermission), en la que las partes exponen su posición y visión del conflicto. Es esencial la utilización de técnicas de escucha activa y desarrollar empatía situacional entre las mismas.

- Fase de recogida de información (Open Doors), en la que el mediador o mediadora recogen lo esencial y relevante de ambas posiciones. Es esencial la utilización de técnicas de escucha activa y desarrollar empatía situacional entre las distintas sensibilidades en juego. Las técnicas y habilidades del medidor son esenciales en esta fase:

○ Preguntar

- Escuchar activamente

- Crear confianza

- Permanecer neutral

- Vínculo comunicacional

- Legitimación de las partes mediante asertividad cognitiva

- Dar empatía

- Parafraseo / Reformulación

- Lenguaje asertivo

En el cuadro siguiente se sintetizan dos de las más importantes habilidades del mediador:

\begin{tabular}{|c|c|}
\hline Cómo escuchar activamente & Cómo activar la empatía \\
\hline $\begin{array}{l}\text { - Con disposición psicológica } \\
\text { ("estar mentalizado") }\end{array}$ & $\begin{array}{l}\text { - Evitando interrumpir } \\
\text { - Evitando emitir juicios de valor }\end{array}$ \\
\hline $\begin{array}{l}\text { "Mediante la técnica de } \\
\text { "observación participante" }\end{array}$ & $\begin{array}{l}\text { - Evitando emitir ayuda prematura ("síndrome } \\
\text { del mediador amigo") } \\
\text { - Evitando juzgar los sentimientos del } \\
\text { interlocutor ("Síndrome del mediador experto") }\end{array}$ \\
\hline $\begin{array}{l}\text { Utilizando de forma } \\
\text { estratégica el lenguaje verbal } \\
\text { y no verbal }\end{array}$ & $\begin{array}{l}\text { O Ofrecer una actitud de empatía proactiva no } \\
\text { significa que estemos de acuerdo con las } \\
\text { actuaciones, pensamientos o posicionamientos del } \\
\text { otro" (Técnica de la "flexibilidad estratégica") }\end{array}$ \\
\hline
\end{tabular}

II. Fase de búsqueda de soluciones (Open Windows), en la que el mediador o mediadora ayuda a las partes al planteamiento de sus posibles soluciones al conflicto.

III. Fase de negociación (Bridge Over), en la que el mediador o mediadora ayuda a acercar las posturas expuestas en la fase anterior, con la finalidad de llegar a una síntesis que conduzca al acuerdo final.

IV. Fase de Acuerdo (Way Out/Way In), en la que el mediador o mediadora redacta el acta final, que si contiene el acuerdo que pone fin a la controversia es firmada por las partes. Si no hubiera sido posible llegar a un acuerdo, solo se redactará el acta final. 
Es usual en las mediaciones transfronterizas o internacionales recurrir a medios telemáticos, mediante la realización de sesiones “on line", utilizando plataformas seguras, en el denominado procedimiento simplificado de mediación a través de medios telemáticos o electrónicos. El resultado, como en el procedimiento ordinario, sigue el mismo modus operandi, aunque al igual que en aquél es muy importante la habilidad y experiencia del mediador o mediadora para acercar posiciones y ayudar proactivamente a alcanzar un acuerdo sólido y robusto que ponga término a la controversia y pacifique la situación preexistente.

\section{LA GAMA DE GRISES}

Es necesario incluir en esta aproximación a la mediación transfronteriza, una expresa reflexión acerca de la interculturalidad en mediación internacional, porque la plena integración social del inmigrante depende, entre otras muchas otras prioridades de amplísimo espectro, pero también de la activación de las ADR como un medio importantísimo para facilitar a través de la resolución de los conflictos interculturales (trans o no transfronterizos) el desarrollo de una vida digna para el ejercicio inmediato o posterior de derechos civiles y políticos. La mediación es un indicador infrautilizado en materia de evaluación de la integración real de los inmigrantes, puesto que, si se implementara de forma sostenida y continua, se podría comprobar como proporciona "feedback" e información inmediata sobre niveles de integración reales y objetivos y ayudaría a entender mejor la realidad de la persona inmigrante que sigue siendo, por definición, un sujeto especialmente vulnerable.

Los mecanismos que la mediación ofrece en la resolución de controversias y conflictos interculturales debería ser en el plano socio-jurídico una conditio sine qua non para promover el libre desarrollo personal de la persona inmigrante, (en relación con sus aspiraciones, expectativas, etc.) como para agilizar su participación en la vida social y contribuir a poner fin, de una vez por todas, al continuo "choque de civilizaciones" que se vierte desde las más arbitrarias posiciones del poder a través de ese intermitente, paralelo y xenófobo discurso cada vez más agresivo, sobre identificación entre inmigración y delincuencia.

La mediación, bien implementada y aplicada, contribuiría poderosamente a la pacificación de muchos conflictos interculturales porque cada vez tenemos más indicadores de resultado que reflejan logros en la realización de derechos a través de la mediación que de la judicialización pura y dura.

\section{BIBLIOGRAFÍA}

ESPLUGUES MOTA, C. (2012). "El régimen jurídico de la mediación civil y mercantil en conflictos transfronterizos en España tras la Ley 5/2012 de 6 de julio", Boletín Mexicano de Derecho Comparado, 136: 165-199.

ORTUÑO MUÑOZ, J. P. y HERNÁNDEZ GARCÍA, J. (2007). "Sistemas alternativos a la resolución de conflictos [ADR]: la mediación en las jurisdicciones civil y penal", Documento de Trabajo, $n^{o} 110$, en http://pascualortuno.wordpress.com.

PALAO MORENO, G. (2013). "Mediación y Derecho Internacional Privado", en Ma E. Vázquez Gómez; $\mathrm{M}^{\mathrm{a}}$ d. Adam Muñoz y N. Cornago Prieto (coords.) El arreglo pacífico de controversias internacionales. Valencia: Tirant lo Blanch, pp. 649-674.

PÉREZ DAUDÍ, V. (2012). "La relación entre la mediación en asuntos civiles y mercantiles y el proceso civil”, Revista de Derecho Mercantil, 283: 231-270. 
SENÉS MOTILLA, C. (2012). "La eficacia del 'compromiso de mediación' y de los 'acuerdos de mediación"”. En LegalToday.com, http://www.legaltoday.com/opinion/articu los-de-opinion/la-eficaciadel-compromiso-de-mediacion-y-de-los-acuerdos-de-mediacion.

\section{Breve currículo:}

\section{Carmelo Hernández Ramos}

Licenciado en Psicología. Diplomado en Criminología. Especialista en Violencia de Género y en Mediación y Justicia Penal. Profesor Docente e Investigador del Departamento de Psicología de la Salud de la Universidad de Alicante, Miembro de los grupos de investigación GITE y PsicoCriminología de la UA. Técnico de la OCI de la Audiencia Provincial de Alicante en medidas alternativas a la prisión (programas de reeducación en violencia de género y doméstica; programas de sensibilización y concienciación en seguridad vial), mediación penal reparadora en adultos (plan piloto del CGPJ) y acompañamiento asistencial e informativo a víctimas de violencia de género. 